\title{
ANALISI ENERGETICA DI CANTIERI DI MECCANIZZAZIONE LEGGERA PER L'UTILIZZAZIONE DI UN CEDUO DI EUCALIPTO
}

\author{
Sanzio Baldini, Rodolfo Picchio, Serena Savelli
}

\section{INTRODUZIONE}

\section{Introduction}

La crisi petrolifera degli anni ' 70 ha incentivato la ricerca nel settore della bioenergia. Diversi progetti vennero iniziati allo scopo di individuare e minimizzare i consumi energetici nei vari settori produttivi, inoltre iniziarono studi approfonditi sulla possibilità di sostituire il petrolio con energie alternative. Sebbene si ottennero buoni risultati e vennero costruite basi promettenti su cui sviluppare futuri progetti, lo stabilizzarsi del prezzo del petrolio e la conseguente regressione della crisi petrolifera, rallentarono i progressi delle ricerche nel campo bioenergetico [16].

Attualmente le fonti energetiche fossili coprono il $76 \%$ del fabbisogno energetico mondiale. Andare verso una società sostenibile implicherebbe un ritorno alle fonti di energia rinnovabili, come l'energia da biomassa. Fino ad ora, le fonti energetiche da biomassa coprono circa $15 \%$ del fabbisogno mondiale. La maggior parte di questa biomassa, $13 \%$, è usata nei paesi in via di sviluppo e soltanto una parte minore, $2 \%$, è usata nei paesi industrializzati [12].

L'attuale riaccentuarsi delle problematiche legate alla disponibilità ed al prezzo del petrolio ed il preoccupante surriscaldamento dell'atmosfera terrestre, ha portato nuovi interessi nel settore della ricerca bioenergetica.

La biomassa come fonte di energia rinnovabile e risorsa con bilancio del carbonio neutro offre interessanti sviluppi per il futuro. Fra le varie biomasse sfruttabili quelle legnose offrono delle buone prospettive sia a livello produttivo che ambientale [16].

Ma le biomasse di origine forestale necessitano per una loro corretta e sostenibile gestione di un'adeguata pianificazione e logistica. In particolare, le utilizza-

Memoria presentata il 22.11.2006; accettata il 10.05.2007

SANZIO BALDINI, Professore ordinario (baldini@unitus.it). RoDOLFO PICCHIO, Ricercatore (r.picchio@unitus.it). SERENA SAVELli, Assegnista di ricerca (s.savelli@unitus.it). D.A.F., Università degli Studi della Tuscia. Il contributo all'impostazione ed allo svolgimento del lavoro va suddiviso tra gli autori in modo paritetico. zioni forestali rappresentano uno degli anelli deboli del sistema, con problematiche organizzative, sociali, economiche ed ambientali.

Con lo scopo di contribuire ad una migliore conoscenza della logistica e dei bilanci energetici dei cantieri di utilizzazione forestale, si riportano i risultati di cantieri di meccanizzazione leggera per l'utilizzazione di un ceduo di eucalipto, tipologia forestale ampiamente diffusa nel sud Italia.

Per l'eucalipto l'unico mercato attualmente sfruttabile è rappresentato dalla legna da ardere per consumo famigliare o per le pizzerie. In questi ultimi anni il legno viene utilizzato in centrali come possibile e valida sorgente di energia, in sostituzione dei combustibili fossili. Proprio nella zona oggetto dello studio sono attualmente in funzione due centrali termoelettriche.

L'utilizzo dei rami e delle chiome nelle zone dove il rischio d'incendio è elevato rappresenta una procedura preventiva importante anche perché il legno dell'eucalipto impiega molti anni prima di decomporsi.

La ricerca ha riguardato il rilievo dei tempi di lavoro distinti per ogni operazione e fase ed il calcolo delle produttività giornaliere ad operaio, dei valori energetici riferiti alle macchine ed attrezzature usate (input indiretti), al consumo di combustibili e lubrificanti (input diretti) e al legname ricavato dall'utilizzazione (output). Il fine è stato quello di utilizzare tali dati per la stesura di un bilancio energetico allo scopo di potere valutare la fattibilità e la convenienza per ogni cantiere e per ogni tipologia di intervento, mantenendo sempre come obiettivo finale quello della produzione di calore dalla biomassa legnosa. Ciò consente non solo l'individuazione dei consumi energetici nelle utilizzazioni dei cedui, ma anche una valutazione dei fabbisogni energetici per diversi metodi produttivi e, al limite, la scelta migliore tra le diverse opzioni di utilizzazione possibili.

\section{MATERIALI E METODI}

\section{Materials and methods}

Il lavoro trattato è stato svolto nei pressi della città 


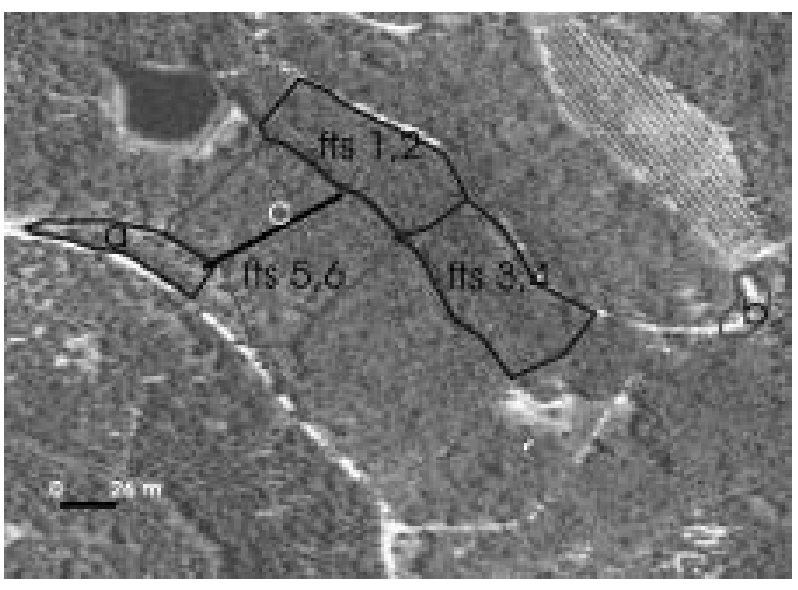

Fig. 1 - Ortofoto dei cantieri. (a: imposto dei cantieri esboscati con gru a cavo; b: imposto dei cantieri esboscati con $i$ verricelli forestali; c: impianto della gru a cavo).

Fig. 1 - Yards orthophoto. (a: tower yard landing; b: winches landing; c: tower yard line).

di Crotone (KR), in località Carpentieri (fig. 1) [1]. Il soprassuolo è costituito da un impianto monospecifico di Eucalyptus occidentalis Endl. di 20 anni che si trova dislocato in zone collinari, caratterizzate da pendenza media prossima al $27 \%$ con picchi massimi per brevi tratti che possono arrivare fino ad un 30-40\%.

Questa specie arborea è largamente diffusa nel sud Italia occupando una superficie, secondo l'inventario forestale nazionale, di 226.400 ha. Scopo iniziale della piantagione era la duplice finalità protettiva e produttiva.

Per lo svolgimento delle prove sperimentali sono state delimitate sei aree, ciascuna della superficie di circa 0,5 ha appartenenti alla II classe di pendenza (20-40\%) e scarsamente accidentate. Dopo aver caratterizzato il popolamento in esame (tab. 1) sono iniziate le operazioni di utilizzazione.

Per l'utilizzazione delle sei aree è stato adottato il sistema della pianta intera (Full Tree System), impiegando mezzi e metodologie di lavoro differenti, in modo da poter valutare la soluzione operativa più idonea da estendersi ai boschi con simili caratteristiche.

Per ogni operazione sono state analizzate le varie fasi di lavoro e rilevati i tempi totali comprensivi dei tempi effettivi di lavoro e dei tempi morti [6] [4] [5].

Successivamente, una volta calcolato il volume della massa legnosa utilizzata, è stato possibile risalire alla produttività oraria, potenziale e reale, di ciascuna operazione.

Gli strumenti utilizzati per l'acquisizione dei dati sono stati: una tabella cronometrica analogica "Minerva", dotata di tre cronometri centesimali, un cavalletto dendrometrico, una cordella metrica autoavvolgente ed un clisimetro per misurare la pendenza del terreno.

In laboratorio si è proceduto alla determinazione della massa volumica e dell'umidità delle piante utilizzate. Per il calcolo di massa volumica ed umidità del legno su due polloni di ogni classe diametrica sono state prelevate delle rotelle. Su ogni pianta scelta sono state prese tre rotelle, una alla base, una a metà lunghezza del fusto ed una all'altezza corrispondente ad un diametro di $3 \mathrm{~cm}$. Da tali rotelle sono stati ricavati i provini, i quali sono stati misurati e pesati allo stato fresco ed allo stato anidro per il calcolo della massa volumica. È stato utilizzato il metodo della doppia pesata. Il volume è stato misurato con un calibro della precisione di $1 / 20 \mathrm{~mm}$. La pesatura è stata effettuata con una bilancia da laboratorio della precisione di 1/1000 di g. Per ottenere il legno anidro è stato impiegato il metodo della essiccazione in stufa a circa $103^{\circ} \mathrm{C}$ per 24 ore.

Partendo dall'analisi dettagliata dei tempi di lavoro e dei quantitativi di legna utilizzati si sono analizzati i cantieri dal punto di vista energetico, per fare ciò ci si è avvalsi del metodo della richiesta lorda d'energia fossile (lubrificanti e combustibili) necessaria per produrre e commercializzare l'unità di massa legnosa.

L'analisi della richiesta lorda di energia (GER, Gross Energy Requirements) è una metodologia appoggiata dall'IFIAS (International Federation of Institutes for Advanced Study, di Stoccolma). Tale analisi ha un approccio pragmatico ai problemi energetici. In essa si sottolinea e si riconosce l'importanza delle fonti energetiche fossili negli attuali sistemi produttivi e soltanto sui flussi di energia fossile (diretta ed indiretta) focalizza il proprio interesse [11]. L'analisi del GER è stata standardizzata mediante opportune convenzioni e definizioni. Il nome "Analisi energetica" è raccomandato per quel particolare campo di ricerca che studia l'energia necessaria nell'approvvigionamento di un bene o un servizio e che risulta così incorporata (sequestrata) in tale prodotto; non solo l'energia diretta impiegata, ma anche quella indiretta associata ai materiali di processo. Per i combustibili è impiegato tradizionalmente il potere calorifico superiore. I valori delle grandezze precedenti possono essere calcolati per unità di prodotto in uscita: o come

\begin{tabular}{|l|r|}
\hline Descrizione & Valore \\
\hline $\mathrm{n}^{\circ}$ ceppaie/ha & 669 \\
\hline $\mathrm{n}^{\circ}$ polloni/ha & 2780 \\
\hline Area basimetrica $\left(\mathrm{m}^{2} / \mathrm{ha}\right)$ & 20,10 \\
\hline Volume $\left(\mathrm{m}^{3} / \mathrm{ha}\right)$ & 120,501 \\
\hline Diametro medio a $1,30 \mathrm{~m}(\mathrm{~m})$ & 0,10 \\
\hline Altezza media $(\mathrm{m})$ & 11,58 \\
\hline Volume medio della pianta $\left(\mathrm{m}^{3}\right)$ & 0,043 \\
\hline Peso medio della pianta tal quale $(\mathrm{kg})$ & 48,7 \\
\hline Peso medio del fusto tal quale $(\mathrm{kg})$ & 39,0 \\
\hline Peso medio dei rami tal quale $(\mathrm{kg})$ & 9,7 \\
\hline Massa volumica tal quale $\left(\mathrm{t} / \mathrm{m}^{3}\right)$ & 1,123 \\
\hline Massa volumica sulla sostanza secca $\left(\mathrm{t} / \mathrm{m}^{3}\right)$ & 0,739 \\
\hline
\end{tabular}

TABElla 1 - Dati produttivi, dendrometrici e tecnologici del popolamento.

TABLE 1 - Dendrometric and technologic crop parameters. 


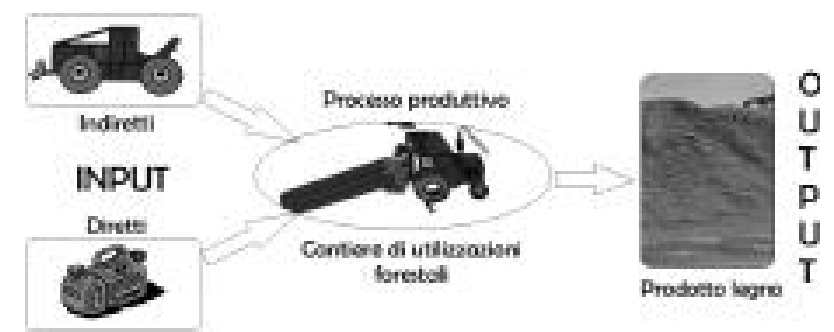

Fig. 2 - Schema del processo produttivo e cicli energetici.

Fig. 2 - Productive and energy cycles diagram.

media (richiesta totale di energia divisa la quantità totale di prodotto in uscita); o come valore marginale (quantità di energia spesa a livello di ultima unità di prodotto ottenuta).

Mettendo in relazione la potenza nominale della macchina $[\mathrm{P}(\mathrm{kW})]$ (tab. 4), il fattore di carico del motore $(\mathrm{Fc}$, in numero assoluto da 0 a 1$)$, il consumo specifico di carburante di un motore [Cs $\left(\mathrm{kg} \mathrm{kW}^{-1} \mathrm{~h}^{-}\right.$ $\left.{ }^{1}\right)$ ] (tab. 4) ed il tempo del suo utilizzo per unità di massa secca prodotta [Tu (h/ts.s.)], siamo riusciti a determinare il consumo unitario di combustibile $\mathrm{Cu}$ $\left(\mathrm{kg} / \mathrm{t}_{\mathrm{s} . \mathrm{s}}\right)$.

$$
\mathrm{Cu}=\mathrm{P} \cdot \mathrm{Fc} \cdot \mathrm{Cs} \cdot \mathrm{Tu}
$$

Per risalire dal valore del consumo unitario (kg/ts.s.) agli input diretti (MJ/t $\mathrm{t}_{\text {s.s. }}$ ) delle macchine impiegate (tab. 4) si sono usate tabelle analitiche che forniscono valori energetici $(\mathrm{MJ} / \mathrm{kg})$ dei principali mezzi di produzione (tab. 2).

Il consumo dei lubrificanti è stato assunto pari a 3 $\mathrm{kg}$ ogni $100 \mathrm{~kg}$ di gasolio consumato e pari ad 1/3 di $\mathrm{kg}$ per ogni $\mathrm{kg}$ di miscela utilizzata [7].

Per il calcolo e la determinazione degli input indiretti delle macchine e delle attrezzature, abbiamo fatto riferimento al valore energetico dei singoli materiali strutturali (tab.3), mettendoli in relazione alla loro presenza quantitativa, il tutto è stato quindi rapportato alla durata tecnica delle macchine, alla loro massa ed al loro effettivo impiego nel cantiere [15]. Tale modo di procedere è stato adottato per le macchine e le attrezzature utilizzate in tutti e sei i cantieri.

Al fine di inquadrare il bilancio energetico nel complesso e formulare dei valori specifici per ogni singolo cantiere riguardo al rapporto "output/input" ed all'efficienza energetica percentuale del sistema,

\begin{tabular}{|l|c|}
\hline \multicolumn{1}{|c|}{ Combustibili } & Input $(\mathbf{M J} / \mathbf{k g})$ \\
\hline Gasolio & 51,5 \\
\hline Benzina & 55,3 \\
\hline Lubrificanti & 83,7 \\
\hline
\end{tabular}

TABELla 2 - Valori dei contenuti energetici per i combustibili ed i lubrificanti impiegati [7].

TABLE 2 - Fuels and lubricants energy contents [7].

\begin{tabular}{|l|r|}
\hline \multicolumn{1}{|c|}{ Materia “prima" } & Contenuto energetico medio (MJ/kg) \\
\hline Materiali ferrosi & 67,5 \\
\hline Materiali non ferrosi & 90,0 \\
\hline Leghe leggere & 360,0 \\
\hline Altri materiali & 112,5 \\
\hline
\end{tabular}

TABELla 3 - Valori dei contenuti energetici delle principali materie prime impiegate [15].

TABLE 3 - The row materials employed energy contents [15].

sono stati determinati gli output. Per la loro determinazione si stima l'energia sprigionata dalla sua combustione [15], quindi è stato preso in considerazione il Potere Calorifico Superiore (P.C.S.) del legno della specie legnosa in esame (Eucalyptus occidentalis Endl.) $19456,5 \mathrm{MJ} / \mathrm{t}_{\mathrm{s} . \mathrm{s}}$.

Premesso che la metodologia (GER) per il calcolo dei bilanci energetici in genere esclude a priori gli input energetici richiesti per il lavoro svolto dagli uomini [7], tuttavia per questo lavoro si è ritenuto utile tentare di porre in bilancio anche gli input relativi al la-

\begin{tabular}{|c|c|c|c|c|c|}
\hline Macchine & $\begin{array}{c}\text { Massa } \\
(\mathbf{k g})\end{array}$ & $\begin{array}{c}\text { Durata } \\
\text { tecnica } \\
\text { (h) } \\
\end{array}$ & $\begin{array}{c}\text { Input } \\
(\mathrm{MJ} / \mathbf{k g})\end{array}$ & $\begin{array}{c}\text { Consumo } \\
\text { specifico } \\
\left(\mathbf{k g ~ k W}^{-1} \mathbf{h}^{-1}\right) \\
\end{array}$ & $\begin{array}{c}\text { Potenza } \\
\text { (kW) }\end{array}$ \\
\hline $\begin{array}{l}\text { motosega di } \\
\text { media potenza }\end{array}$ & 6 & 2000 & 152,1 & 0,35 & 3,2 \\
\hline $\begin{array}{l}\text { trattore agricolo } \\
\text { gommato } \\
\text { versione } \\
\text { forestale } \\
\text { da } 60 \mathrm{~kW}\end{array}$ & 5000 & 10000 & 92,4 & 0,25 & 60 \\
\hline $\begin{array}{l}\text { trattore agricolo } \\
\text { gommato } \\
\text { da } 98 \mathrm{~kW}\end{array}$ & 5800 & 10000 & 106,1 & 0,25 & 98 \\
\hline $\begin{array}{l}\text { verricello } \\
\text { forestale a } 1 \\
\text { tamburo } \\
\text { da } 40 \mathrm{kN} \\
\end{array}$ & 300 & 5000 & 71,3 & & \\
\hline $\begin{array}{l}\text { verricello } \\
\text { forestale a } 2 \\
\text { tamburi } \\
\text { da } 40 \mathrm{kN} \\
\end{array}$ & 500 & 5000 & 71,3 & & \\
\hline $\begin{array}{l}\text { impianto } \\
\text { completo di } \\
\text { verricello per } \\
\text { gru a cavo } \\
\end{array}$ & 600 & 8000 & 83,7 & & \\
\hline $\begin{array}{l}\text { Sminuzzatrice } \\
\text { biber } 7\end{array}$ & 2000 & 6000 & 72,5 & & \\
\hline $\begin{array}{l}\text { Sminuzzatrice } \\
\text { bruks CT900 }\end{array}$ & 3700 & 6000 & 83,9 & 0,25 & 263 \\
\hline $\begin{array}{l}\text { Autocarro per } \\
\text { sminuzzato }\end{array}$ & 7500 & 10000 & 121,5 & 0,25 & 310 \\
\hline $\begin{array}{l}\text { Autocarro per } \\
\text { tronchetti }\end{array}$ & 8500 & 10000 & 121,2 & 0,25 & 310 \\
\hline
\end{tabular}

TABELLA 4 - Tabella analitica delle macchine impiegate, per il calcolo degli input energetici.

TABLE 4 - Analytic table of the employed machines, for the energy input calculation. 
voro umano. Tutto ciò non è stato di semplice svolgimento, mostrando complicazioni che in questo primo lavoro sono state solo marginalmente superate.

Il conteggio energetico del lavoro umano ha dato luogo negli anni ad un esteso dibattito affrontato dai più diversi punti di vista. Da una parte i fautori di un calcolo di equivalenza energetica delle ore di manodopera (per esempio la scuola eco-energetica di H.T. Odum); dall'altra, gli oppositori con le più diverse motivazioni. In linea di principio è evidente che il lavoro muscolare ha una sua quantificazione come potenza ed energia, ma è altrettanto evidente che una valutazione energetica del lavoro umano descrive in maniera estremamente limitata e riduttiva le ore di manodopera impiegate in un processo produttivo [7].

Tuttavia, la posizione dell'IFIAS [10] sul problema del lavoro umano è la seguente: "ove l'analisi (energetica) si riferisca ad economie sviluppate o industrializzate non è necessario considerare l'energia necessaria per lo stile di vita della manodopera. Ove l'analisi consideri agricolture non intensive la valutazione della manodopera gioca un ruolo importante nei calcoli" [7], ritenendo il settore forestale nella fattispecie i cantieri esaminati ricadenti all'interno della sopra citata tipologia, il computo energetico del lavoro umano si ritiene assuma la sua importanza.

I calcoli sono stati sviluppati sulla base di una ricerca svolta valutando l'impegno fisico dei lavoratori forestali in base a misure del consumo di ossigeno, della ventilazione polmonare e della frequenza cardiaca. Da questa ricerca è emerso un valore medio (comprendente le diverse mansioni svolte da chi esegue le utilizzazioni forestali) di consumo energetico nell'unità di tempo pari a $50,2 \mathrm{~kJ}$ min $^{-1}$ operaio $^{-1}(12 \mathrm{kcal}$ min $^{-1}$ operaio $^{-1}$ ) [13].

Questi dati pur se rilevati in ambienti alpini, nel corso di operazioni svolte su fustaie, sono al momento una valida base di partenza che ci permette di quantificare, seppur con approssimazione, l'apporto energetico del lavoro umano nei cantieri analizzati, inoltre il valore ricavato corrisponde anche a quanto citato da Hippoliti [9], il quale individua una media giornaliera totale di 17600-21800 kJ gg-1 operaio $^{-1}$ (circa 4200$5200 \mathrm{kcal} \mathrm{gg}^{-1}$ operaio $^{-1}$ ).

In ciascuna delle sei aree si è impiegato lo stesso sistema di utilizzazione a pianta intera (FTS), ma con una diversa meccanizzazione, una diversa organizzazione del cantiere ed un prodotto all'imposto differente (fig. 1).

In ogni area si è proceduto all'abbattimento direzionato dei polloni tramite una motosega di media potenza Husqvarna 357XP ed al concentramento manuale dei polloni abbattuti in fastelli, la squadra era composta da 3 operatori.

fts 1 e 2 - L'esbosco è stato fatto con un verricello forestale a un tamburo Krpan con forza di tiro di 40 $\mathrm{kN}$ applicato all'attacco a tre punti di un trattore agricolo a ruote, versione forestale FIAT 780 DT della potenza di $60 \mathrm{~kW}$, la squadra era composta da 2 operatori. Nel corso di questa operazione si sono rilevati valori medi di distanze per lo strascico indiretto di 58,3 m e per lo strascico diretto di 89,8 m.

fts 3 e 4 - L'esbosco è stato fatto con un verricello forestale a due tamburi Maxwald con forza di tiro di $40 \mathrm{kN}$ per ciascun tamburo, applicato all'attacco a tre punti di un trattore agricolo a ruote, versione forestale FIAT 780 DT della potenza di $60 \mathrm{~kW}$, la squadra era composta da 3 operatori. Nel corso di questa operazione si sono rilevati valori medi di distanze per lo strascico indiretto di $23,1 \mathrm{~m}$ e per lo strascico diretto di $76,7 \mathrm{~m}$.

fts 5 e 6 - L'esbosco è stato fatto mediante una gru a cavo leggera (fig. 3) con stazione motrice basata su un verricello forestale a un tamburo Krpan con forza di tiro di $40 \mathrm{kN}$ applicato all'attacco a tre punti di un trattore agricolo a ruote, versione forestale FIAT 780 DT della potenza di $60 \mathrm{~kW}$, la squadra era composta da 4 operatori. Nel corso di questa operazione si sono rilevati valori medi di distanze per lo strascico laterale di $15,1 \mathrm{~m}$ e per il semistrascico di $48,8 \mathrm{~m}$.

fts 1, 3 e 5 - All'imposto i polloni esboscati sono stati sramati e depezzati in tronchetti lunghi $2 \mathrm{~m}$ con una motosega identica a quella impiegata nell'abbattimento, quindi la ramaglia veniva ammucchiata ed $\mathrm{i}$ tronchetti accatastati manualmente, la squadra era composta da 6 operatori.

fts 2, 4 e 6 - All'imposto i polloni esboscati sono stati sminuzzati con una sminuzzatrice a tamburo Biber 7 ad alimentazione manuale (fig. 4), azionata da un trattore agricolo a ruote New Holland TS 130 della potenza di $98 \mathrm{~kW}$, la squadra era composta da 4 operatori.

Il materiale all'imposto era differenziato a seconda del tipo di cantiere che lo aveva prodotto, sottoforma di tronchetti da $2 \mathrm{~m}$ nel caso dei cantieri "fts $1,3,5$ " e sottoforma di scaglie nel caso dei cantieri "fts $2,4,6$ ", con destinazione d'uso produzione di energia, presso un'industria di utilizzazione finale posta a circa $20 \mathrm{~km}$ dal cantiere. Onde poter concludere l'intero ciclo del prodotto, fino al piazzale di stoccaggio presso l'impresa di utilizzazione finale, si sono ipotizzate due si-

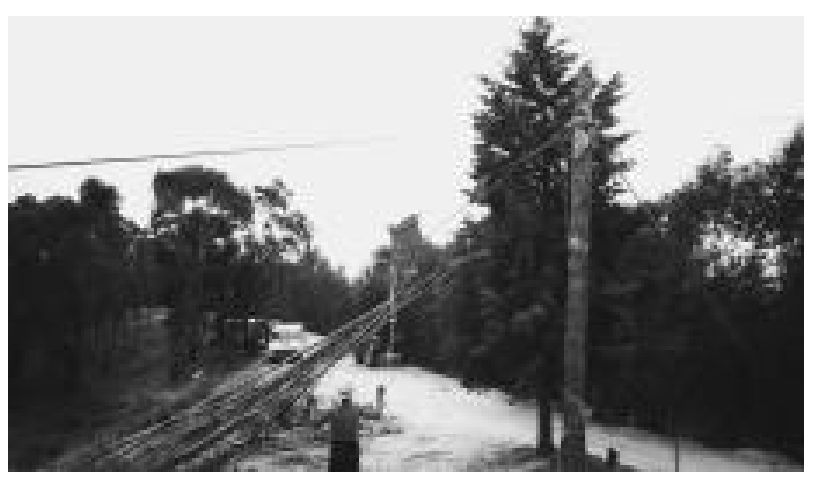

Fig. 3 - Zona di scarico del materiale dall'impianto di gru a cavo, ritto di monte e carrello.

Fig. 3 - Downloading zone of the light tower yard, superior standing and carriage. 


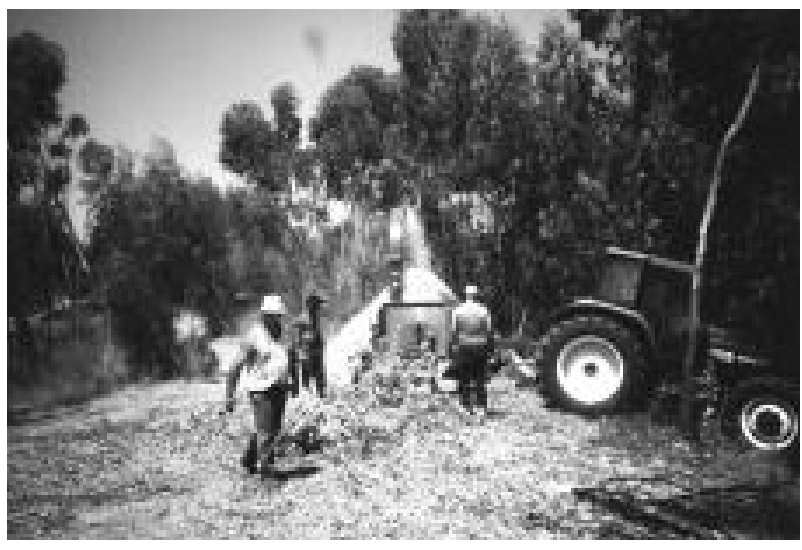

Fig. 4 - Sminuzzatura con alimentazione manuale.

Fig. 4 - Chipping with manual feed.

tuazioni tecnicamente fattibili ed economicamente valide, corredate da idonei dati di bibliografia.

Nel caso dei tronchetti da $2 \mathrm{~m}$ il trasporto è programmato in funzione della tipologia di percorso da affrontare tramite un autocarro a tre assi con portata massima di $16 \mathrm{t}$, cassone senza sponde equipaggiato con stanti per il trasporto dei tronchi e munito di gru idraulica per il carico e lo scarico. La produttività ed i tempi di lavoro della fase di carico sono stati reperiti attraverso un'indagine bibliografica [2]. Inoltre questo materiale una volta giunto al piazzale verrà trasformato in scaglie impiegando una sminuzzatrice a tamburo con motore autonomo e alimentazione meccanica di notevoli dimensioni, anche in questo caso da un'indagine bibliografica abbiamo ricavato valori di produttività e tempi di lavoro ritenuti idonei al contesto in cui ci trovavamo [14].

Nel caso delle scaglie l'unica mancanza era il trasporto, anche in questo caso si è programmato in funzione della tipologia di percorso da affrontare tramite un autocarro a tre assi con portata massima di $17 \mathrm{t}$, cassone con sponde rialzate, ribaltabile su tre lati. In questo caso il carico avveniva direttamente attraverso il convogliatore pneumatico della sminuzzatrice.

Per entrambi gli autocarri vista la tipologia di strade da percorrere, circa $3 \mathrm{~km}$ di pista camionabile e 17 $\mathrm{km}$ di viabilità ordinaria in discrete condizioni di cui $2 \mathrm{~km}$ inizio centro abitato, si è risaliti ad una velocità media di percorrenza di $50 \mathrm{~km} / \mathrm{h}$ [9].

\section{RISULTATI E CONSIDERAZIONI CONCLUSIVE}

\section{Results and conclusive considerations}

Da un'analisi dei dati ottenuti, relativi al popolamento (tab. 1), alle produttività medie lorde ed all'impiego della manodopera, si evince come nel complesso la metodologia di lavoro più idonea sembra essere quella della sminuzzatura direttamente all'imposto della pianta intera ed il relativo trasporto all'utenza fi-

\begin{tabular}{|l|c|c|c|c|c|c|}
\hline \multicolumn{1}{|c|}{ Cantieri } & fts 1 & fts 2 & fts 3 & fts 4 & fts 5 & fts 6 \\
\hline $\begin{array}{l}\text { Impiego } \\
\text { manodopera } \\
\text { [h/t s.s.] }\end{array}$ & 7,35 & 4,80 & 7,50 & 4,95 & 9,03 & 6,47 \\
\hline $\begin{array}{l}\text { Impiego } \\
\text { manodopera } \\
\text { [gg/ha] }\end{array}$ & 81,98 & 53,52 & 83,65 & 55,20 & 100,66 & 72,20 \\
\hline $\begin{array}{l}\text { Produttività } \\
\text { media reale } \\
{\left[\mathrm{t}_{\text {s.s. }} \mathrm{h}^{-1}\right.} \\
\text { operaio }^{-1} \text { ] }\end{array}$ & 0,14 & 0,21 & 0,13 & 0,20 & 0,11 & 0,15 \\
\hline
\end{tabular}

TABELla 5 - Dati relativi all'impiego di manodopera ed alle produttività medie, nel processo di produzione delle scaglie. Valori considerati fino allo stabilimento di utilizzo finale.

TABLE 5 - Data summary of human employment and of average productivity, in the chips production. Cautious values until the final utilization industrial plant.

nale delle scaglie (tab. 5, fts 2,4,6).

Per quanto riguarda invece il livello di meccanizzazione impiegato, soprattutto riguardo al concentramento ed esbosco, sempre considerando i livelli di produttività riscontrati, sembra sia la tipologia meno meccanizzata ad ottenere il miglior risultato, in questo caso il trattore con verricello forestale ad un tamburo (tab. 5, fts 1 e 2). Certo rispetto al trattore equipaggiato con verricello forestale a due tamburi le differenze sono veramente minime in questo caso (tab. 5 , fts 3 e 4). L'impiego dell'impianto di gru a cavo leggera, invece pur avendo fornito buoni livelli di produttività (tab. 5, fts 5 e 6), in considerazione anche della presenza di pendenze medie inferiori al $40 \%$ e per distanze medie di esbosco prossime ai $50 \mathrm{~m}$ (quanto riscontrato nei cantieri), non è consigliabile.

Analizzando i risultati relativi all'analisi energetica, si riscontra una differenza tra gli output rilevati nei cantieri dove è stata effettuata la sminuzzatura della pianta intera (tab. 6 , fts $2,4,6$ ) e quelli dove sono stati

\begin{tabular}{|l|c|c|c|c|c|c|}
\hline \multicolumn{1}{|c|}{ Cantieri } & fts 1 & fts 2 & fts 3 & fts 4 & fts 5 & fts 6 \\
\hline $\begin{array}{l}\text { Output } \\
\text { (GJ/ha) }\end{array}$ & 1387 & 1736 & 1387 & 1736 & 1387 & 1736 \\
\hline $\begin{array}{l}\text { Input diretti } \\
\text { ed indiretti } \\
\text { dei cantieri } \\
\text { (GJ/ha) }\end{array}$ & 160,2 & 155,0 & 152,0 & 146,7 & 156,3 & 151,0 \\
\hline $\begin{array}{l}\text { Input lavoro } \\
\text { umano } \\
\text { (GJ/ha) }\end{array}$ & 2,0 & 1,3 & 2,0 & 1,3 & 2,4 & 1,7 \\
\hline $\begin{array}{l}\text { Input totale } \\
\text { (GJ/ha) }\end{array}$ & $\mathbf{1 6 2 , 2}$ & $\mathbf{1 5 6 , 3}$ & $\mathbf{1 5 4 , 0}$ & $\mathbf{1 4 8 , 0}$ & $\mathbf{1 5 8 , 7}$ & $\mathbf{1 5 2 , 7}$ \\
\hline
\end{tabular}

TABELla 6 - Dati relativi all'analisi energetica per singola tipologia di cantiere, riferiti all'unità di superficie del soprassuolo forestale (ha), valori considerati fino allo stabilimento di utilizzo finale.

TABLE 6 - Energy analysis data for individual typology of yard, reported to the forest area (ha), cautious values until the final utilization industrial plant. 
allestiti tronchetti lunghi $2 \mathrm{~m}$ (tab. 6, fts 1,3,5). Questo è spiegabile per il fatto che nella seconda tipologia, a differenza della prima, la ramaglia veniva ammucchiata e bruciata all'imposto, mentre la biomassa che entrava nel ciclo di utilizzo finale era solo quella rappresentata dal fusto della pianta.

Gli input diretti per ogni singolo cantiere rappresentano in media circa il 95\% del totale, mentre gli indiretti costituiscono in media il $5 \%$ e dove viene computato anche il lavoro umano questa suddivisione si scosta di poco, in quanto il lavoro umano in media rappresenta poco più dell' $1 \%$ del totale degli input, almeno con la metodologia di calcolo seguita. Senza per altro far riscontrare rilevanti differenze fra un cantiere e l'altro.

I cantieri che hanno fatto riscontrare i consumi energetici intesi come carburanti (input diretti) più elevati sono fts 1 e 2 dove l'esbosco è stato eseguito con trattore e verricello forestale a un tamburo. Mentre per quanto concerne gli input indiretti ovvero imputabili alle macchine ed attrezzature impiegate, $i$ più dispendiosi sono stati i cantieri fts 5 e 6 dove si è impiegato per l'esbosco l'impianto di gru a cavo leggera. Lo stesso può dirsi circa il lavoro umano espresso in termini energetici.

Andando a considerare ora i bilanci nel complesso, sia in termini di rapporto fra output ed input, sia in termini di efficienza energetica percentuale, il risultato non cambia, il cantiere che garantisce la maggiore efficienza è fts 4 , dove si è impiegato per l'esbosco il trattore con verricello forestale a due tamburi; un buon risultato è stato fornito anche nel caso di impiego dell'impianto di gru a cavo leggera, mentre il trattore con verricello forestale ad un tamburo ha fornito il risultato peggiore.

Ragionando in termini di tipologia di meccanizzazione, senza alcun dubbio la maggior efficienza energetica è garantita dai cantieri dove si è sminuzzata la

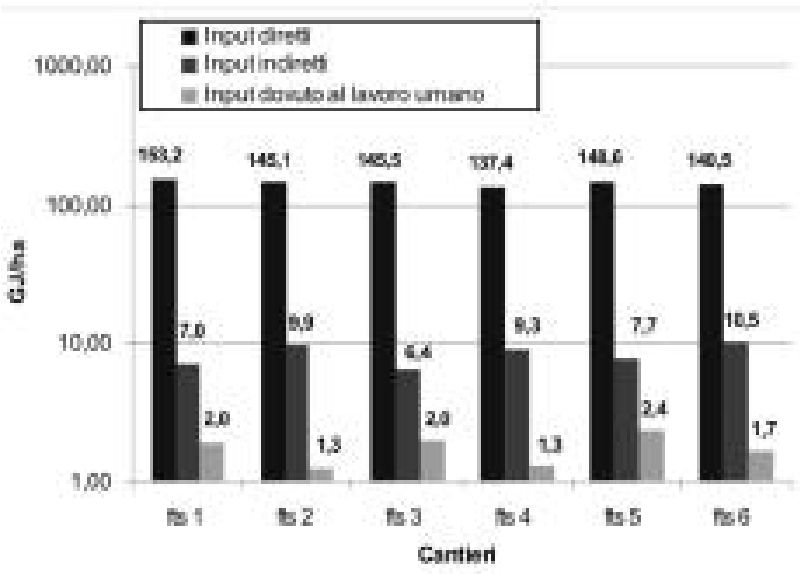

Fig. 5 - Istogramma dei valori degli input energetici diversificati per cantiere e per tipologia. Valori considerati fino allo stabilimento di utilizzo finale.

Fig. 5 - Histogram of the energy input values diversified for yard and for typology. Cautious values until the final utilization industrial plant.

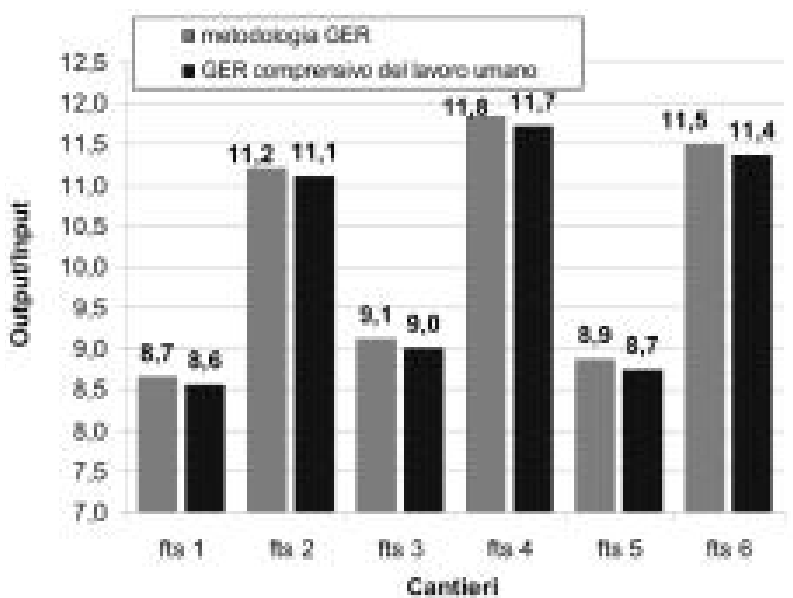

Fig. 6 - Istogramma dei valori di bilancio energetico (Output/Input) per singolo cantiere di utilizzazione. Valori considerati fino allo stabilimento di utilizzo finale.

Fig. 6 - Histogram of the energy values balance (Output/Input) for individual utilization yard. Cautious values until the final utilization industrial plant.

pianta intera all'imposto.

Interessante è anche notare come per i casi studiati tra l'impiego di manodopera sul cantiere e l'efficienza energetica del cantiere vi sia un rapporto inverso infatti i cantieri con maggior efficienza energetica sono stati anche quelli che hanno richiesto il minor impiego di manodopera (fig. 8).

Infine analizzando nel complesso i dati ricavati, soprattutto considerando i livelli di produttività reale, l'impiego di manodopera ed i valori di bilancio energetico si può affermare come i cantieri di maggior interesse siano quelli dove si è eseguita la sminuzzatura della pianta intera all'imposto (fts $2,4,6$ ) ed entrando ancor più nel dettaglio il livello di meccanizzazione più indicato è quello che prevede l'esbosco con tratto-

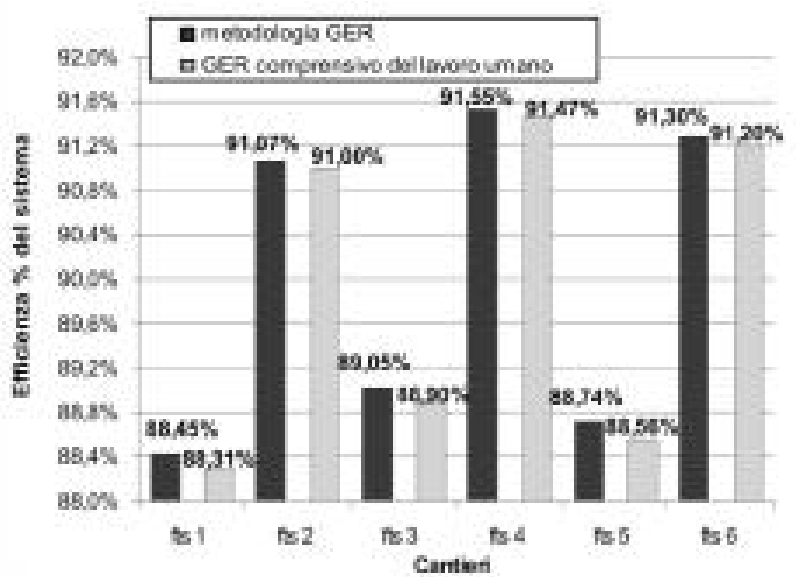

Fig. 7 - Istogramma che esprime l'efficienza energetica percentuale [(Output-Input)/Output*100] per singolo cantiere. Valori considerati fino allo stabilimento di utilizzo finale.

Fig. 7 - Histogram that expresses the energy efficiency percentage [(Output-Input)/Output*100] for individual yard. Cautious values until the final utilization industrial plant. 


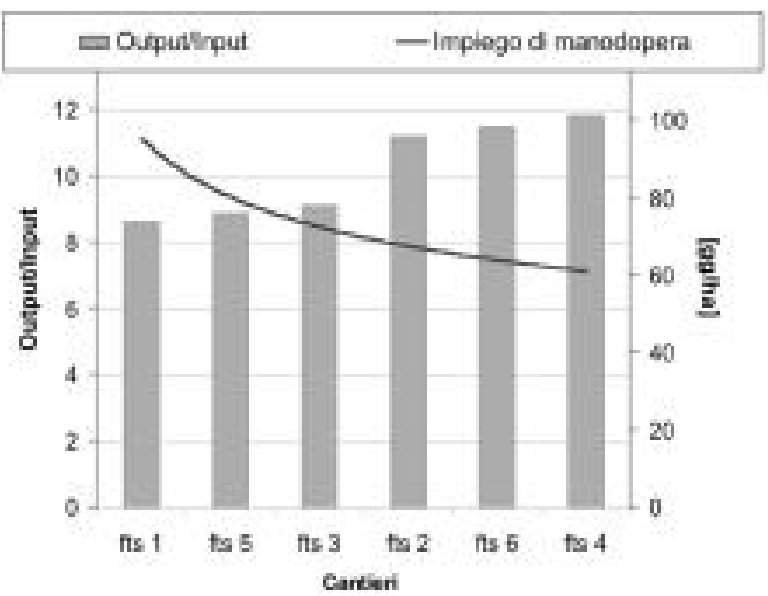

Fig. 8 - Grafico che evidenzia come per i casi studiati l'andamento del bilancio energetico eseguito con metodologia GER sia inverso a quello dell'impiego di manodopera. Anche in questo caso $i$ valori sono differenziati per singola tipologia di cantiere di utilizzazione, e riferiti all'intero processo di produzione delle scaglie ad uso energetico. Valori considerati fino allo stabilimento di utilizzo finale.

Fig. 8 - Graph that emphasizes like for the studied cases the tendency of energy balance performed with GER methodology is opposite to that of the man employment. Also in this case the values are differentiated for individual yard. Cautious values until the final utilization industrial plant.

re e verricello forestale a due tamburi (fts 4) o in alternativa quello con trattore e verricello forestale ad un tamburo (fts 2), questi due cantieri in effetti si alternano avendo il primo l'optimum energetico ed il secondo il primato in fatto di produttività.

\section{BIBLIOGRAFIA}

\section{References}

[1] Baldini S., Bernabei M., Picchio R., Harvesting of Eucalyptus plants with simple methods and analysis of the chips obtained, atti del Convegno internazionale "Eucalyptus in the Mediterranean Basin: Perspectives and new utilisations", Taormina (2000), 87-109.

[2] Baldini S., Gallo P., Picchio R., Produttività, sicurezza e qualità, fattori a confronto nell'utilizzazione di una fustaia, Sherwood (2006), 123 (6), 41-47.

[3] Baldini S., Picchio R., Ruocco T., Analisi delle produttività e dei bilanci energetici in tre cantieri di utilizzazione, atti del VII Convegno nazionale A.I.I.A. "Ingegneria agraria per lo sviluppo dei Paesi Mediterranei", Vieste (2001), CD-rom.

[4] Baldini S., Pollini C., Proposta europea di protocolli armonizzati per lo studio del lavoro in bosco, Sherwood (2000), 56 (5), 43-45.

[5] Baldini S., Pollini C., Proposta europea di protocolli armonizzati per lo studio del lavoro in bosco, Sherwood (2000), 58 (7), 45-47.

[6] Berti S., Piegai F.,Verani S, Manuale di istruzione per il rilievo dei tempi di lavoro e delle produttività nei lavori forestali, Quaderni dell'Istituto di Assestamento e Tecnologia Forestale dell'Università di Firenze, (1989), IV, 65.
[7] Biondi P., Panaro V., Pellizzi G., Le richieste d'energia del sistema agricolo italiano, Progetto finalizzato energetica (1989), 79.

[8] CARTER A.P., Applications of input-output analysis to energy problems, Science (1974), 184 (4134), 325-329.

[9] Hippoliti G., Appunti di meccanizzazione forestale (1997), Studio Editoriale Fiorentino, Firenze.

[10] IFIAS, Energy Analysis, workshop report n ${ }^{\circ} 6$, Stoccolma, (1974).

[11] IFIAS, Energy Analysis and Economics, workshop report $n^{\circ} 9$, Stoccolma (1975).

[12] Johansson J., LuNDQVisT U., Estimating Swedish biomass energy supply, Biomass and Bioenergy (1999) 17, 85-93.

[13] Rodio A., Rosponi A., Fattorini L., Delussu S., MarchetTi E., MARChetTI M., Costo energetico durante attività forestali in ambiente alpino, atti del convegno "Lavoro in foresta e salute", Trento (2002), 172-181.

[14] Spinelli R., Hartsough B., Indagini sulla cippatura in Italia, CNR-CSP XLI, (2001), Firenze.

[15] VolPI R., Bilanci energetici in agricoltura (1992), Laruffa Editore, Reggio Calabria.

[16] Yoshiokaa T., Arugab K., Nitamic T., Sakaid H., KoBayashic H., A case study on the costs and the fuel consumption of harvesting, transporting, and chipping chains for logging residues in Japan, Biomass and Bioenergy (2006) 30,342-348.

\section{Parole chiave:}

biomassa forestale, utilizzazioni forestali, bilancio energetico, eucalipto.

\section{SUMMARY}

\section{ENERGY ANALYSIS OF LIGHT MECHANIZA- TION YARDS FOR THE EUCALYPTUS COP- PICE HARVEST}

The work was turned near Crotone (KR), in a 20 year-old plantation of Eucalyptus occidentalis Endl. positioned in hill zones, with average slope next to $27 \%$.

The utilisation system was the Full Tree System (FTS). The felling on the six yards has been done by chainsaw and the first coppice bunching was manual.

fts 1,2 - The extraction has been done by one drum forest winch applied to the three point hitch of a wheeled farm tractor forest version.

fts 3,4 - The extraction has been done by two drums forest winch, applied to the three point hitch of a wheeled farm tractor forest version.

fts 5,6 - The extraction has been done by light tower yarder powered by one drum forest winch applied to the three point hitch of a wheeled farm tractor forest version.

fts 1, 3 and 5 - At the landing the coppice have been processed by chainsaw in 2 meters length trunks. The slash accumulated and the trunks staked manually.

fts 2, 4 and 6 - At the landing the coppice have been chipped by drum chipper with manual feed. The machine was a tractor powered chipper. 
At the landing the wood has been differentiated in two types. The type from the yards "fts $1,3,5$ " were 2 meters length trunks and the other type from the yards "fts 2,4,6" were chips for energy production. For the 2 meters length trunks the transport has been done by truck with $16 \mathrm{t}$ of load capacity, and hydraulic crane. This material at the staple will be chipping by drum chipper with autonomous engine and mechanical feed. The chips transport will be done by truck with $17 \mathrm{t}$ of load capacity.

From the working times analysis and the wood quantitative obtained we studied the energy balance in the yards. We used the GER (Goss Energy Requirement) method. From the results obtained, the best work methodology seems to be that of full trees chip- ping directly at the landing and the chips transport at the staple.

For the bunching and the extraction the best productivity result is given from the tractor with one drum forest winch. Considering the energy balance the yard that guarantees the greater efficiency is the "fts 4", where has been employed for the extraction the tractor with two drums forest winch. The mechanization typology that supplied the better energy efficiency is that of the yards "fts 2,4 and 6" where the full trees has been chipped to the landing.

\section{Key words:}

Forestry byomass, forestry utilisation energy balance, eucalyptus. 